\title{
Microbiological Aspects in Biofilm Produced by some Uropathogens Isolated from Patients with Indwelling Bladder Catheters
}

\author{
Mushtak T. Salih Narjis F. AL-Ani \\ Department of Microbiology \\ College of Medicine \\ University of Al-Anbar
}

(Received 22/ 8 / 2011 ; Accepted 26/12/2011)

\begin{abstract}
Seventy five catheter urine specimens obtained from sixty four patients and admitted to Urology and other departments in Ramadi Teaching Hospital were studied. qualitative and quantitative detection for biofilm production for all isolates were achieved. Antimicrobial susceptibility test for planktonic cells of study isolates was performed by broth dilution method and the MICs were detected. Further, In Vitro model for formation of pseudomonal biofilm on catheter segments by biofilm producer-isolates of the same bacteria was achieved. BICs and MBECs were detected for all sessile study isolates of Pseudomonas aeruginosa against ciprofloxacin, amikacin and cefotaxime.

Out of 75 catheter urine specimens, 61 (81.4\%) were positive for culture including: Escherichia coli 17 (27.9\%) followed by Klebsiella spp. 13 (21.3\%), Pseudomonas aeruginosa 12 (19.7\%), and in spectrophotometric method, the results showed that all of 12 isolates of Pseudomonas aeruginosa, 7 isolates of Proteus mirabilis, 17 isolates of Escherichia coli and 13 isolates of Klebsiella, 12 (100\%), 7 (100\%), 12 (70.6\%) and 12 (92.3\%) isolates produced biofilm in the glucose supplemented media. In biofilm antimicrobial susceptibility, the sessile study isolates of Pseudomonas aeruginosa were required 10-50 X MICs from ciprofloxacin and amikacin to inhibit bacterial biofilm and 50-100 X MICs to eradicate it from the surfaces of catheter in contrast to cefotaxime of which BICs were from 50-100 X MICs and MBECs from 100-1000 X MICs.

The study concluded that Escherichia coli was the most common uropathogen followed by Klebsiella spp., and Pseudomonas aeruginosa.. Also, all study isolates of Pseudomonas aeruginosa and Proteus mirabilis produced biofilm qualitatively and quantatively. Further, with regard to ciprofloxacin and amikacin, the study isolates were also required 10-50 X MICs to inhibit bacterial biofilm and 50-100 X MICs to remove pseudomonal adhesion from the catheters while those for cefotaxime were 50-100 X MICs and 100-1000 MICs for inhibition and eradication of bacterial biofilm respectively.
\end{abstract}

Keywords: Bacterial biofilm, Catheterized UTI, BICs, MBECs. 


\section{مقالق إحيائية مجهربة اللغشاء الحيوي المنتج من الممرضلت البولية والمعزولةمن المرض حالي أنلبيب قظلرة المثلة}

\section{الملغص}

عزلت البكتريا من 75 عينة ادرار مستحصلة من أنابيب قططرة المثانة تعود ل 64 مريض خخل ـوا

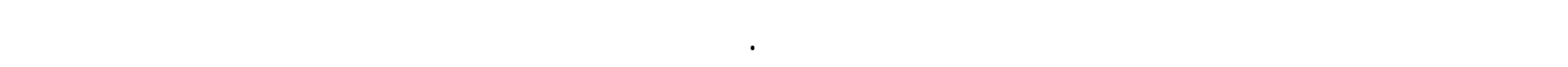

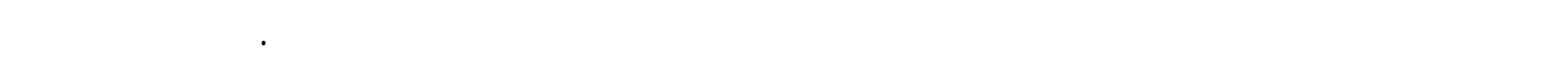

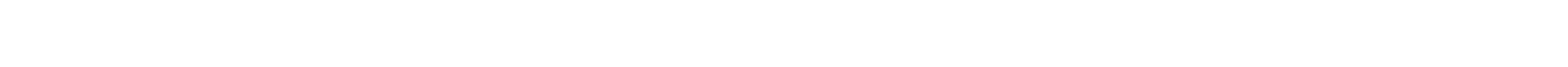

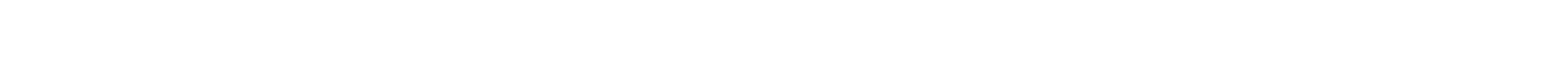

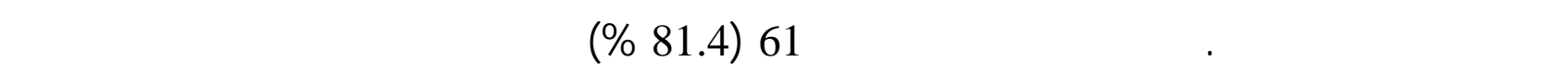

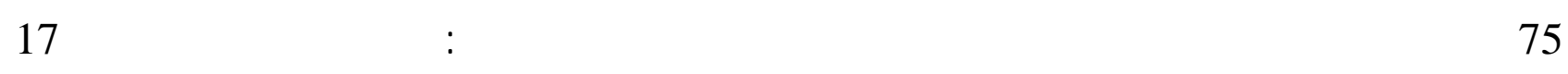

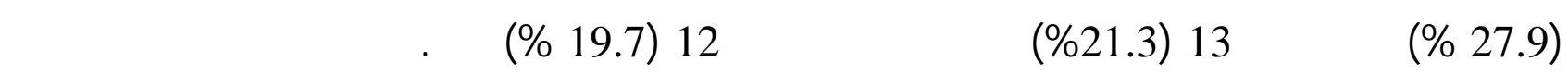

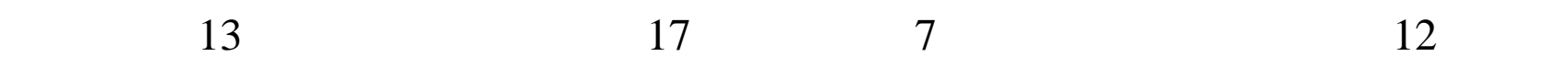

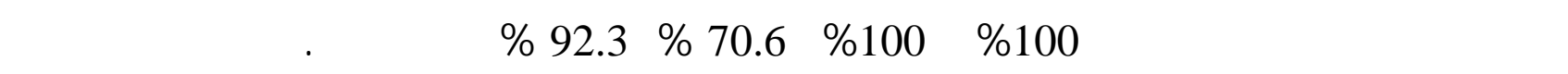

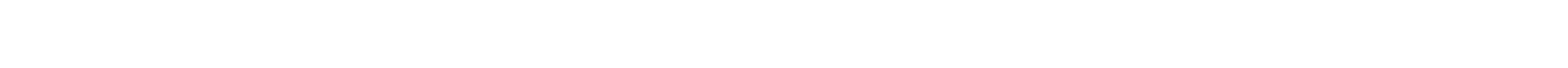

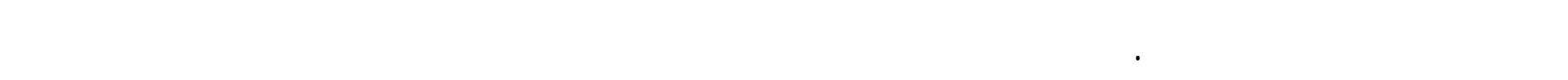

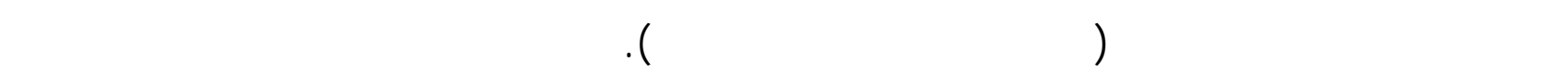

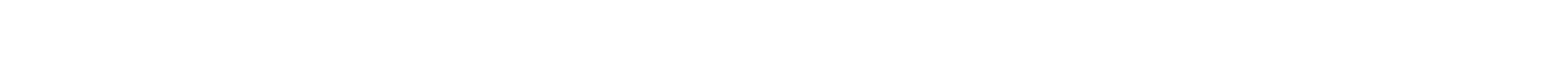

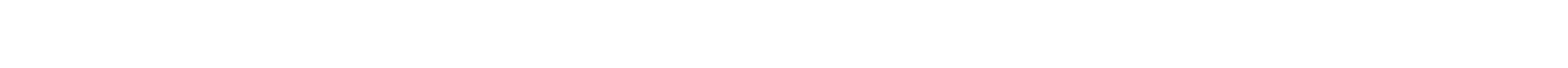

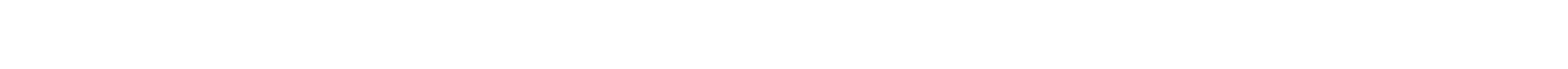

\section{INTRODUCTION}

A biofilm in its nature is bacteria enclosed within exopolysaccharide. It is well realized that infection remains a major obstacle to the long-term use of many implanted medical devices such as indwelling bladder catheters (Katsikogianni and Missirlis, 2004). These indwelling catheters predispose patients to UTIs; in a time that catheterization became indispensable in almost all fields of medicine (Von-Eiff et al., 2005). Urinary catheters are usually tubular latex (siliconized) devices, which when inserted may readily acquire biofilms on the inner or outer surfaces. The organisms commonly contaminating these devices and developing biofilms are Escherichia coli, Klebsiella pneumoniae, Pseudomonas aeruginosa, Proteus mirabilis and other gram-negative organisms (Stickler, 1996). Certain 
organisms of these biofilms produce urease, which hydrolyzes urea in the patient's urine to ammonium hydroxide, the elevated $\mathrm{pH}$ results in precipitation of minerals. These mineralcontaining biofilms form encrustations and consecutive obstruction that may completely block the inner lumen of the catheter ( Donalan, 2002). Bacteria may ascend the inner lumen into the patient's bladder in 1 to 3 days; this rate may be influenced by the presence of swarming organisms such as Proteus spp. (Donalan, 2001). These infections may be complicated by reaching to the kidney causing pyelonephritis (Jones et al., 2005).

Bacterial biofilms are inherently resistant to antimicrobial agents in addition to host immunity (Costerton et al., 1999). In Vitro biofilms often require from 10 to 1,000-fold the concentration of antibiotic to be eradicated as compared to planktonic bacteria. Often the MIC concentration of antibiotic, which by definition is effective against the planktonic cells, was not effective against biofilm cultures of the same organism (Olson et al., 2002). Due to importance of biofilm related infections, the treatment is performed by prolonged and highdose of antimicrobial therapy as well as elimination of infected medical device are the cornerstones of a successful therapy (Fux et al., 2003). New susceptibility parameters are introduced to clinical practice like biofilm inhibitory concentration (BIC) and minimal biofilm eradication concentration (MBEC). Thus, this study has been undertaken to shed a light on the practical points of bacterial biofilm in more details and in several directions, firstly In Vitro, To detect biofilm formation by some uropathogens isolated from patients with indwelling bladder catheters qualitatively and quantitatively by spectrophotometric assay with ELISA reader. Further, in terms of MICs and sub-MICs, to determine antimicrobial susceptibility test for logarithmic phase planktonic cells of study isolates of Pseudomonas aeruginosa. Further more, in term of BICs and MBECs, to detect biofilm antimicrobial susceptibility test for biofilm producer isolates of Pseudomonas aeruginosa to selected antimicrobial agents.

\section{Patients Study}

A total of seventy five (75) catheter urine specimens belonged to sixty four (64) patients admitted to Department of Urology in Ramadi General Hospital was studied during the period from May to September 2007. In some cases, two urine catheter specimens obtained for patient's follow up. Catheter specimens of urine (CSU) were obtained by withdrawing a sample with a syringe and needle from the catheter tube (junction area between catheter and collection tube). The duration for catheter for most patients was more than 7 days (Mims et al., 1993).

Firstly, urine specimen was cultured immediately by semiquantitative culture technique. In this technique, under aseptic conditions a standardized (fixed known volume) inoculum of uncentrifuged urine were streaked on the sectors of nutrient, blood and MacConkey agar plates and incubated at $37^{\circ} \mathrm{C}$ for 24 hours, if no growth was detected, plates were re-incubated for another 24 hours before reported as negative cultures. After incubation, the total number of colonies per ml was counted. Afterthat, the specimen was examined macroscopically and microscopically. All microbiological investigations were also confirmed by using biochemical tests (Baron et al., 1994). 


\section{Biofilm Study}

\section{-Qualitative assay of biofilm formation:}

A- Tube method: (Adhesion assay):

Glycocalyx production was determined as described by (Yassien and Khardori, 2001); (Mathur et al., 2006) as the following:

1. Two to three colonies of study isolate were inoculated into $5 \mathrm{ml}$ of brain heart infusion broth supplemented with $2 \%$ glucose in plastic conical tubes.

2. Cultures were incubated at $37^{\circ} \mathrm{C}$ for $18-24$ hours.

3. The contents were aspirated; one tube was examined unstained and other stained with safranin or crystal violet.

4. Slime positivity was judged by the presence of visible stained or unstained film lining the wall of the tube.

\section{B- Formation of biofilm on catheter:}

An overnight culture of tested bacteria $(10 \mu \mathrm{l})$ in brain heart infusion broth was inoculated into 500 $\mathrm{\mu l}$ of the same medium and injected into clear Foley catheters (the silicon type Foley catheter was used). The catheters were capped at both ends and incubated at $37^{\circ} \mathrm{C}$ overnight. Then catheters were rinsed with phosphate buffer saline. After drying at room temperature for $15 \mathrm{~min}, 700 \mu \mathrm{l}$ crystal violet (1\%) was added to the catheters for 20 min, then the stained biofilm rinsed several times with BPS and allowed to dry at room temperature before examination (Mireles et al., 2001); (Arif, 2006).

\section{Quantitative assay of biofilm formation (Microtiter plate assay)}

Adhesion and biofilm formation was determined by using a spectrophotometric method described by (Yassien and Khardori, 2001); ( Stepanovic et al., 2003) as the following:

1. Working cultures were prepared by inoculation study isolate on Columbia agar supplemented with 5\% blood and incubated aerobically at $35^{\circ} \mathrm{C}$ for $24 \mathrm{hr}$.

2. These cultures were used to prepare standardized bacterial suspension in sterile normal saline adjusted to a $0.5 \mathrm{McF}$ arland turbidity standard to reach $10^{8} \mathrm{CFU} / \mathrm{ml}$.

3. The suspensions obtained were inoculated into a brain heart infusion broth with glucose (glucose supplemented medium) and without glucose.

4. $200 \mu \mathrm{L}$ of standardized cultures were added to each well of sterile polystyrene Microtiter plate and incubated at $37^{\circ} \mathrm{C}$ for $18 \mathrm{hr}$.

5. Following incubation, the content of each well was aspirated, and each well was washed 3-4 times with sterile distilled water.

6. The remaining attached bacteria were fixed with $200 \mu \mathrm{L}$ of methanol per well.

7. After 15 minutes, the plate wells were emptied and left to air dry.

8. Afterwards the plates were stained for $5 \mathrm{~min}$ with $160 \mu \mathrm{L}$ per well of crystal violet (0.25\%).

9. Excess stain was rinsed off by placing the Microtiter plate under the running tap water.

10. The plates were air dried and the dye which was bound to the adherent cells was resolubilized with $160 \mu \mathrm{L}$ of $33 \%$ glacial acetic acid per well. 
11. Finally the optical density (OD) of each well was measured at 570 and $630 \mathrm{~nm}$ by using Stat Fax 3200 Elisa Reader.

The isolates were classified according to biofilm production depending on the criteria laid down by Christensen et al., (1985) as following: Strong producer more than 0.240; weak producer between 0.125-0.250 while non-producer less than 0.120.

\section{Antimicrobial susceptibility for planktonic cells}

Broth macrodilution method (MIC method):

Three antimicrobial agents used for planktonic and biofilm study belonged to the following groups: Ciprofloxacin (flouroquinolones), Amikacin (aminoglycosides), and Cefotaxime (third generation cephalosporins). Seven study isolates of Pseudomonas aeruginosa were included in this part of study. The bacterial standardization was performed according to 0.5 McFarland turbidity standards (Miles and Amyes, 1996). Different antibiotic concentrations $(0.5-256 \mu \mathrm{g} / \mathrm{ml})$ were prepared in $5 \mathrm{ml}$ of Mueller-Hinton broth and transferred to sterile capped tubes. At least 4-5 morphologically similar colonies were inoculated into Mueller-Hinton broth and incubated at $37^{\circ} \mathrm{C}$ until the viable turbidity was equal to the $0.5 \mathrm{McF}$ arland, (about $10^{8} \mathrm{cfu} / \mathrm{ml}$ ). After that, the suspension was diluted 1:100 and certain volumes transferred to the tubes containing antibiotic dilutions, to reach a final cell concentration of (about $10^{5} \mathrm{cfu} / \mathrm{ml}$ ). Negative controls were represented by two tubes; one of them contained broth only and the other contained broth plus microorganism. Then the tubes were incubated overnight at $37^{\circ} \mathrm{C}$. The result of minimal inhibitory concentration (MIC) was interpretated as the lowest concentration of antimicrobial agents which inhibits visible bacterial growth after overnight incubation (Ferraro et al., 2000).

\section{Biofilm antimicrobial susceptibility test \\ Formation of biofilm producer-isolates of Pseudomonas aeruginosa on catheter segments (In vitro model):}

The method used for bacterial biofilm formation on catheter segments was described by (Ishida et al., 1998). Briefly, the tested bacteria incubated in brain heart infusion broth overnight at $37^{\circ} \mathrm{C}$. Then $10 \mu \mathrm{L}$ of overnight culture was added to $500 \mu \mathrm{L}$ of sterile media in which catheter segments $\left(1 \mathrm{~cm}^{2}\right)$ was inoculated, and subsequently incubated overnight at $37^{\circ} \mathrm{C}$. After that washing for the segments was achieved by sterile media (3-4) times to remove weakly attached bacteria. Then segments were resuspended with sterile media and vortexed vigorously for 2 min which was considered as controls.

\section{Bactericidal activity of antibacterials against biofilm forming sessile cells}

To test the bactericidal activity of selected antibiotics against the sessile cells, the catheter segments was incubated with the organism as described above, were taken out, washed gently with sterile media or saline and subsequently transferred to saline containing a given antibiotic with distinct concentrations (10X, 50X, 100X, 500X and 1000X) at which $\mathrm{X}$ represent the minimal inhibitory concentration of mentioned antimicrobial agents against planktonic cells which was previously detected. After that the tubes were incubated for 24 hr. at $37^{\circ} \mathrm{C}$ (Arif, 2006). 


\section{Extraction and quantification of biofilm bacteria}

After exposure of tested organism to the desired concentrations of antibiotic, they were transferred to $10 \mathrm{ml}$ of fresh brain heart infusion broth and stirred vigorously with a vortex mixer for $2 \mathrm{~min}$. for dispersion sessile or adherent cells. Then the suspension was diluted and plated on nutrient agar plates for bacterial colony counting and compared with original bacterial count before exposuring to antimicrobial agents (Ishida et al., 1998).

\section{Detection of biofilm inhibitory concentrations (BICs) and minimal biofilm eradication concentration (MBECs):}

After incubation the tubes for $24 \mathrm{hr}$. at $37^{\circ} \mathrm{C}$, the biofilm inhibitory concentration was detected and defined as the lowest concentrations of antimicrobial agents which inhibits bacterial biofilm growth on a surface of catheter. It was represented by the clearance of broth medium consisting $(1 \mathrm{~cm})$ catheters and the required concentrations of antimicrobial agents (Ceri et al., 2006). After plating the diluted suspension into agar plates and counting the number of bacterial colonies, minimal biofilm eradication concentration (MBEC) was determined. MBEC was defined as the lowest concentration of antibiotic or biocide capable of killing biofilm producer bacteria. It was represented by disappearing of colonies of biofilm producer organisms on the culture plates (Ceri et al., 2006).

\section{RESULTS}

Out of 75 catheter urine specimen, 61 (81.4\%) were culture positive, 7 (9.3\%) sterile urine while the other 7 (9.3\%) were defined as sterile Pyuria. Among 61 (81.4\%) culture positive cases, the yielded microbial causative agents were distributed as the followings: Escherichia coli 17 (27.9\%), Klebsiella spp. 13 (21.3\%), Pseudomonas aeruginosa 12 (19.7\%), Staphylococci 9 (14.8\%) divided into 4 (44.4\%) Staphylococcus aureus and 5 (55.6\%) Staphylococcus epidermidis, Proteus mirabilis 7 (11.5\%), Enterobacter spp. 1 (1.6), in addition to Candida spp which was found as a mixed form with the above mentioned bacteria in 2 (3.3\%) cases.

Under the field of biofilm production, particularly qualitative biofilm assay, tube method, our result showed that out of 17 (27.9\%) isolates of Escherichia coli, 13 (21.3\%) isolates of Klebsiella species, 12 (19.7\%) isolates of Pseudomonas aeruginosa, and 7 (11.5 \%) Proteus mirabilis, biofilm were produced on the inner lining of the tubes in 11 (64.7\%), 9 (69.2\%), 12 (100\%), and 7 (100\%) respectively. On the other hand this phenomenon was not observed in 6 (35.3 \%), 4 (30.8\%) and 1 (100\%) of Escherichia coli, Klebsiella spp. and Enterobacter spp. respectively.

Regarding formation of biofilm on catheters, After incubation of the catheter which was previously inoculated with an over night culture of $P$. aeruginosa, a clear biofilm covering the catheter lumen was detected after staining with $1 \%$ crystal violet.

Further, in the other qualitative assay (foley catheter method) our study revealed that out of 17 isolates of Escherichia coli, 13 isolates of Klebsiella species, 12 isolates of Pseudomomnas aeruginosa, and 7 Proteus mirabilis, biofilm were produced on the surface of the catheters in 11 (64.7\%), 11 (84.6\%), 12 (100\%), 7 (100\%) respectively. Furthermore, biofilm production was not observed in 6 (35.3\%), 2 (15.4\%) and 1 (100\%) of Escherichia coli, Klebsiella spp. and Enterobacter spp. respectively. 


\section{Quantitative biofilm production (Spectrophotometric assay)}

In the quantitative biofilm formation assay, spectrophotometric method was achieved under two set of experimental conditions (with and without glucose).

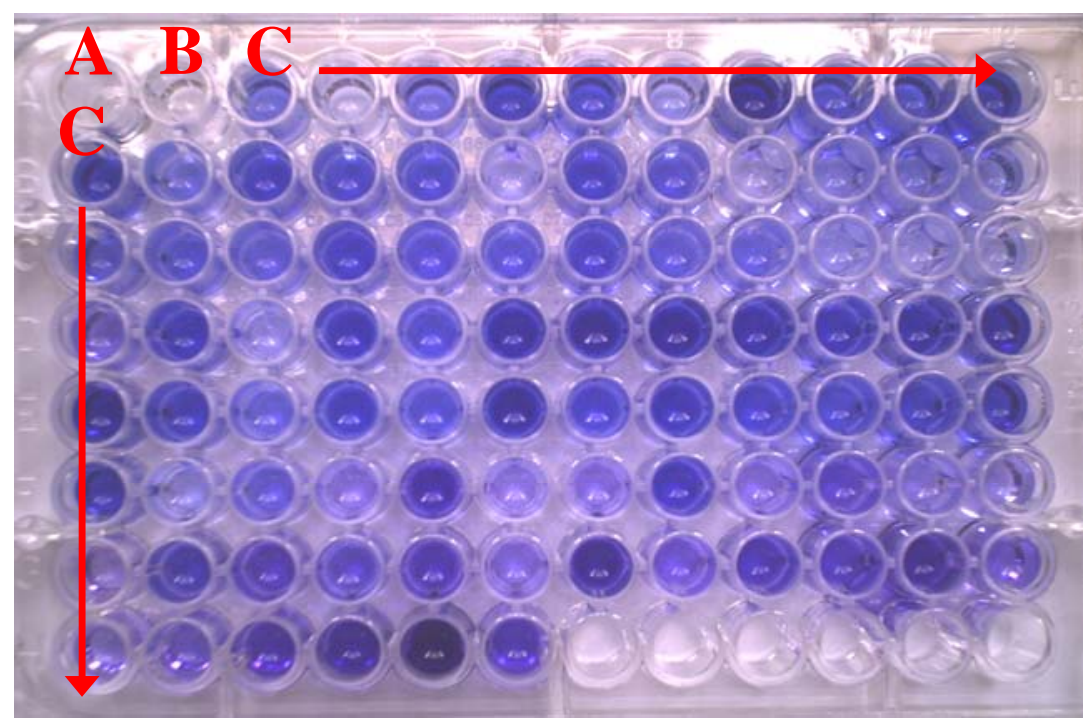

Fig. 1: Biofilm formation on microtiterplate wells (A: Air blank (empty), B: control (brain heart infusion only), C: study isolates).

Our results showed that out of 17 isolates of Escherichia coli, 12 (70.6\%) were biofilm producers. All of these isolates were strong biofilm producers (OD was more than 0.25) with the presence of glucose in the experiment. Further, with the absence of glucose 12 (70.6\%) out of 17 isolates of Escherichia coli produced biofilm weakly. Nine (75\%) of these were strong biofilm producers while 3 (25\%) of them produced biofilm weakly (OD was between 0.125 and 0.25 ). On the other hand no significant differences observed in the readings of optical density at $570 \mathrm{~nm}$ with the presence and absence of glucose among isolates of Escherichia coli $(0.366 \pm 0.335)$ and $(0.351 \pm 0.282)$ respectively ( $\mathrm{P}$ value $=$ 0.886) (Fig. 2).

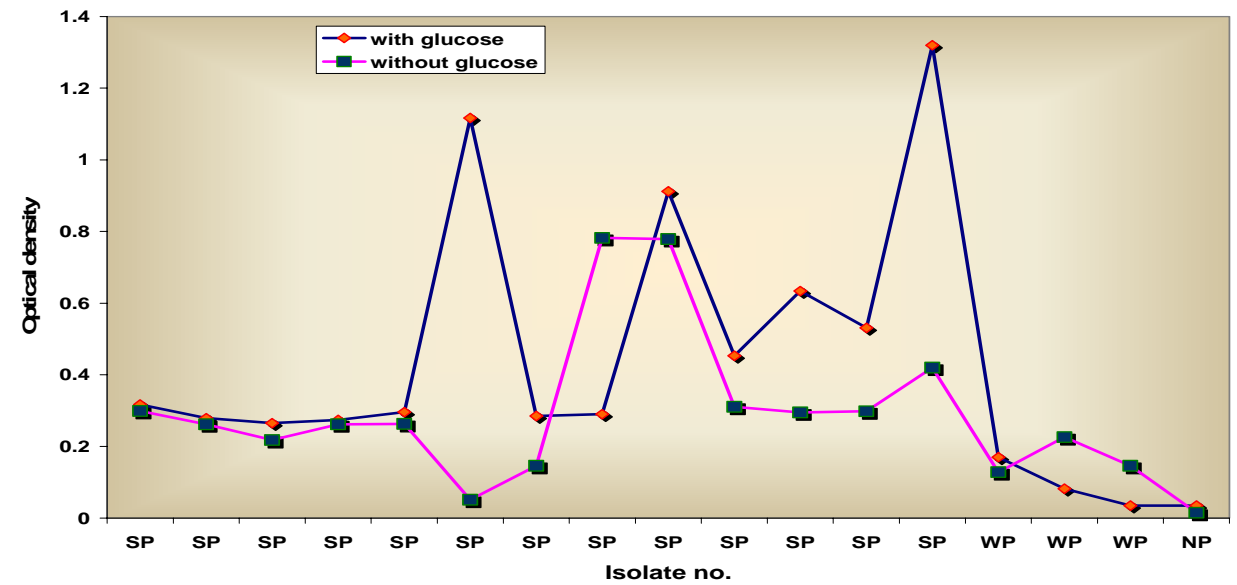

Fig. 2: Effect of glucose on the production of biofilm by spectrophotometric assay among strong and weak biofilm producer isolates of Escherichia coli. (SP:Strong biofilm producer, WP:- Weak producer, NP:- Non biofilm producer). 
Also, our study revealed that out of 13 isolates of Klebsiella spp., 12 (92.3\%) of them were biofilm producers in the glucose supplemented media. Of these, 10 (83.3\%) Klebsiella isolates were produced biofilm strongly, while $2(16.7 \%)$ isolates weakly produced biofilm. On the other hand in the absence of glucose, $11(84.6 \%)$ out of 13 isolates produced biofilm of them 7 (63.6\%) strong biofilm producers and 4 (36.4\%) weak biofilm producers. Also, no significant differences were observed in the readings of optical density at $570 \mathrm{~nm}$ with the presence and absence of glucose among isolates of Klebsiella spp. (0.746 \pm 0.564$)$ and $(0.598 \pm 0.538)$ respectively $(\mathrm{P}$ value $=0.502)($ Fig. 3$)$.

Further, our results appeared that all study isolates of Pseudomonas aeruginosa, 12 (100\%) were produced biofilm in the glucose supplemented media. Of these, 11 (91.7\%) isolates were produced biofilm strongly while 1 (8.3\%) isolates produced biofilm weakly. On the other hand, in the absence of glucose, 10 (83.3\%) out of 12 isolates were produced biofilm of them 7 (70\%) were strong biofilm producers and 3 (30\%) were weak biofilm producers. Also, there is statistically significant differences in the reading of optical density at $570 \mathrm{~nm}$ with the presence and absence of glucose among isolates of Pseudomonas aeruginosa $(0.78 \pm 0.62)$ and $(0.36 \pm 0.22)$ respectively ( $\mathrm{P}$ value $=0.037)$ (Fig. 4).

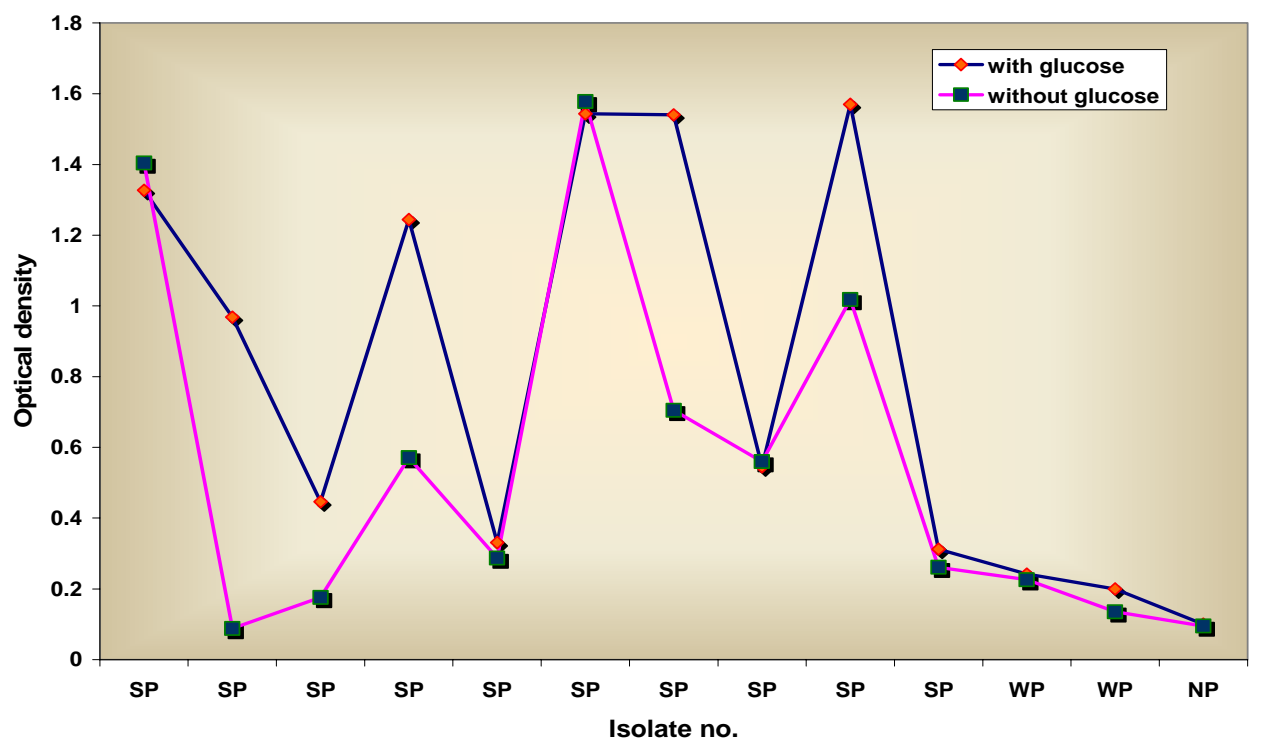

Fig. 3: Effect of glucose on the production of biofilm by spectrophotometric assay among strong and weak biofilm producer isolates of Klebsiella spp. (SP:Strong biofilm producer, WP:- Weak producer, NP:- Non biofilm producer). 


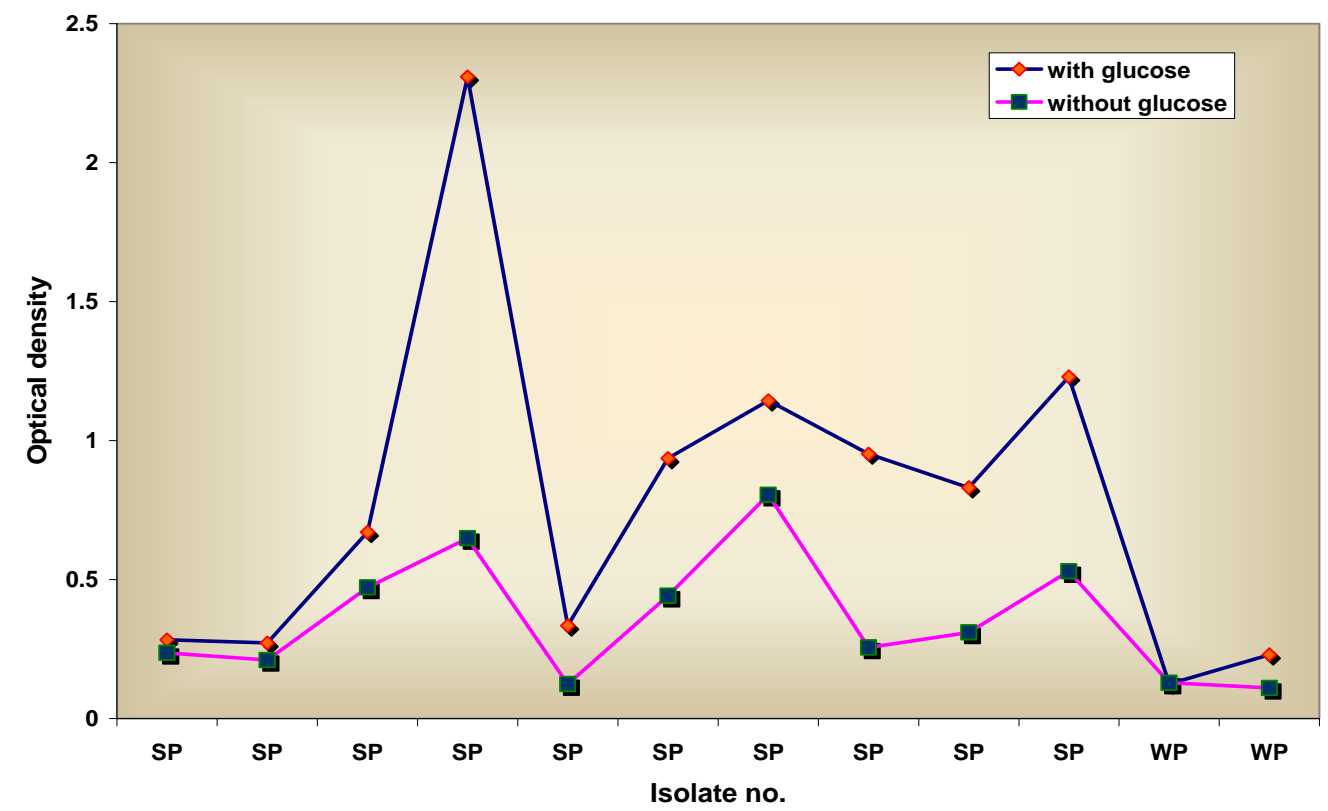

Fig. 4: Effect of glucose on the production of biofilm by spectrophotometric assay among strong and weak biofilm producer isolates of Pseudomonas aeruginosa. (SP:-Strong biofilm producer, WP:-Weak producer, NP:-Non biofilm producer).

Also, our study revealed that all study isolates of Proteus mirabilis produced biofilm in the glucose supplemented and non-supplemented media, of these $6(85.7 \%)$ produced biofilm strongly while 1 (14.3\%) isolates were weakly biofilm producers under two mentioned conditions respectively. Further, the readings means of optical density at $570 \mathrm{~nm}$ with the presence and absence of glucose among isolates of Proteus mirabilis were 1.233 \pm 0.511 and $0.754 \pm 0.363$ respectively ( $\mathrm{P}$ value $=0.067)$ (Fig. 5 ).

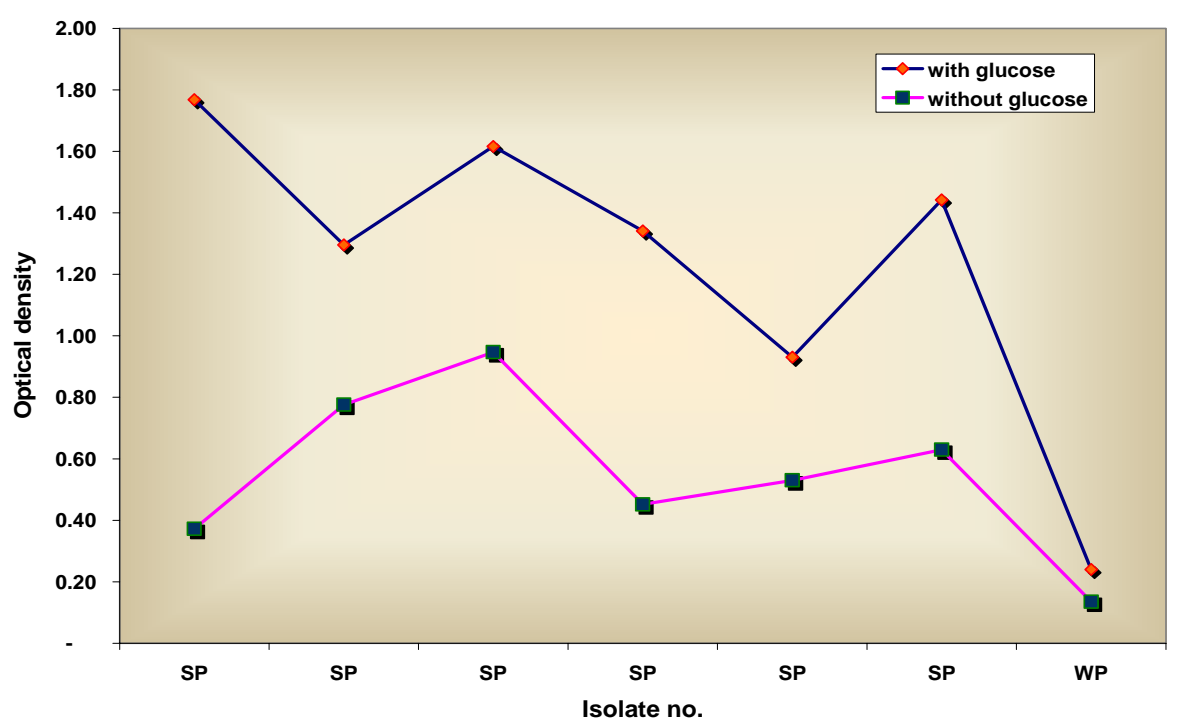

Fig. 5: Effect of glucose on the production of biofilm by spectrophotometric assay among strong and weak biofilm producer isolates of Proteus mirabilis. (SP:Strong biofilm producer, WP:-Weak producer, NP:-Non biofilm producer). 


\section{Antimicrobial susceptibility test for planktonic cells}

\section{-Broth macrodilution technique:}

In this part of study, our result showed that minimal inhibitory concentrations were $26.29 \pm 10.03,118.86 \pm 24.19$ and $10.29 \pm 3.90$ for ciprofloxacin, cefotaxime and amikacin respectively against logarithmic phase planktonic cells of Pseudomonas aeruginosa while the sub-minimal inhibitory concentrations were $13.14 \pm 5.01,59.43 \pm 12.09$ and $5.14 \pm 1.95$ for the same antimicrobial agents respectively.

\section{Biofilm antimicrobial susceptibility test}

Antimicrobial susceptibility test for sessile cell of Pseudomonas aeruginosa was achieved by detecting the biofilm inhibitory concentration (BIC) and minimal biofilm eradication concentration (MBEC). Concentrations of 1, 10, 50, 100 and $500 \mathrm{X}$ MIC were chosen to determine the biofilm inhibitory concentrations (BICs) and minimum biofilm eradication concentrations (MBECs).

With regard to ciprofloxacin, the BIC for N4, N6, N10, N12, N15, N26 and N30 study isolates were 320 (10 XMIC), 320 (10 XMIC), 1600 (50 XMIC), 320 (10 XMIC), 320 (10 XMIC), 1600 (50 XMIC), 320 (10 XMIC) $\mu \mathrm{g} / \mathrm{ml}$ respectively. These isolates has been shown clearance of broth (inhibition of biofilm) and reduced the viable count of bacterial biofilm from $38 \times 10^{5}, 30 \times 10^{5}, 79 \times 10^{5}, 113 \times 10^{5}, 160 \times 10^{4}, 85 \times 10^{5}$, and $67 \times 10^{5}$ to $45 \times 10^{2}, 37 \times 10^{2}, 72$, 96, $33 \times 10^{2}, 34 \times 10^{2}$ and $63 \times 10^{2}$ respectively. On the other hand, the concentrations of 1600 (50 XMIC), 1600 (50 XMIC), 3200 (100 XMIC), 1600 (50 XMIC), 1600 (50 XMIC), 3200 (100 XMIC), 1600 (50 XMIC) $\mu \mathrm{g} / \mathrm{ml}$ were enough to eradicate pseudomonal biofilm from the catheters for the above mentioned isolates respectively with high significant difference, MIC mean $(26.29 \pm 10.028)$ and MBEC mean (1942.86 \pm 907.11 ) with P value < 0.0001 (Fig. 6).

Third generation cephalosporins, cefotaxime inhibited pseudomonal biofilm at the concentrations range between 50-100 folds higher than MIC at the following inhibitory concentrations: 12800, 12800, 6400, 6400, 3200, 1600, and $1600 \mu \mathrm{g} / \mathrm{ml}$ for the above mentioned isolates of Pseudomonas aeruginosa respectively. (Fig. 7).

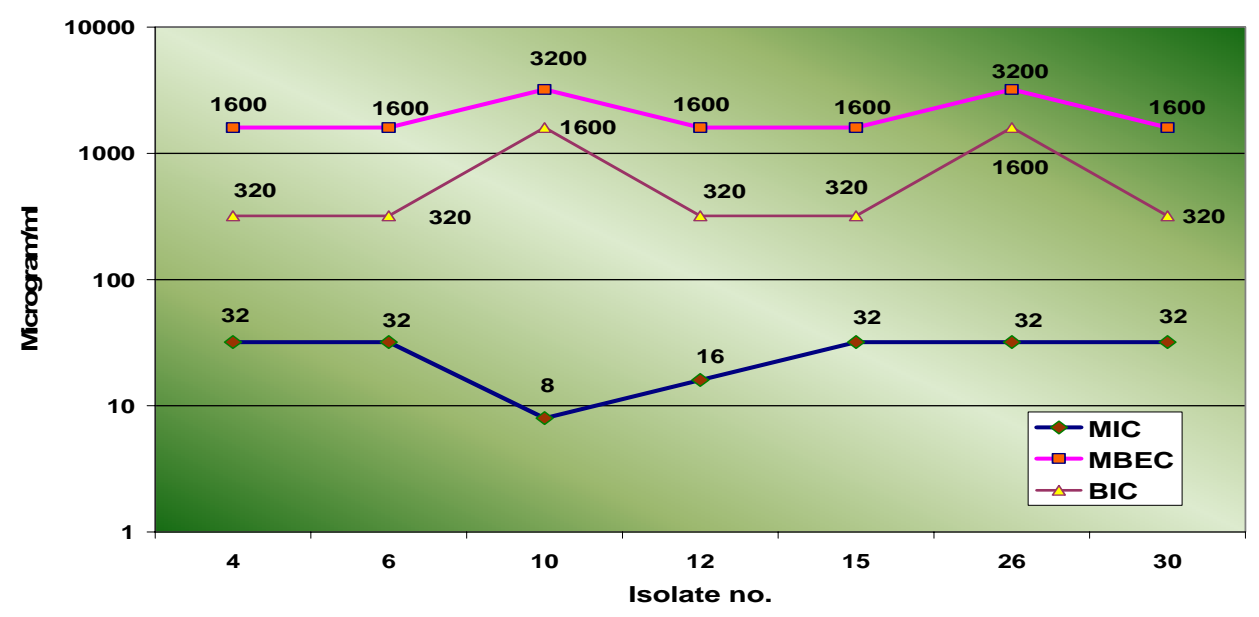

Fig. 6: Comparison of susceptibility parameters for ciprofloxacin against logarithmic phase planktonic cells and sessile (adherent) cells of seven study isolates of Pseudomonas aeruginosa. 


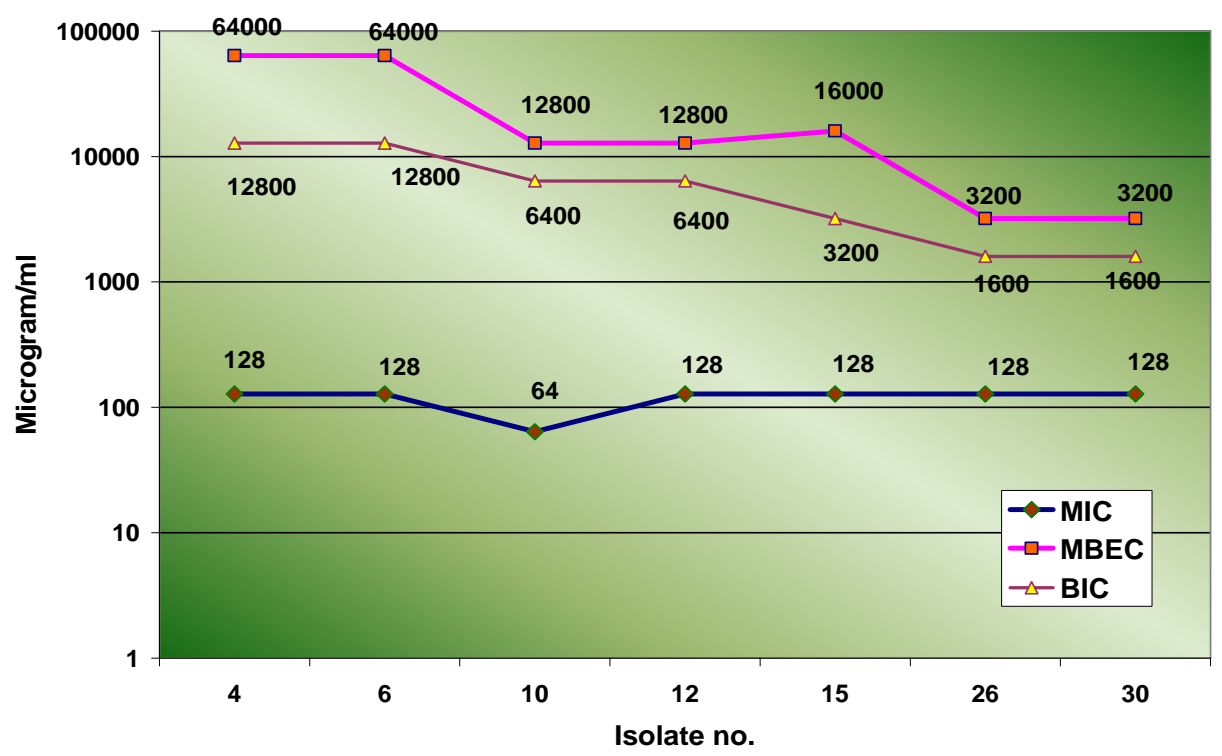

Fig. 7: Comparison of susceptibility parameters for cefotaxime against logarithmic phase planktonic cells and sessile (adherent) cells of Seven study isolates of Pseudomonas aeruginosa.

Also, our results showed that amikacin, inhibit pseudomonal biofilm at the following inhibitory concentrations: 800, 1600, 800, 80, 400, 80, and $400 \mu \mathrm{g} / \mathrm{ml}$ for the above mentioned isolates of Pseudomonas aeruginosa respectively and reduced the viable counts for these isolates markedly as observed in table 4.10, but these concentrations were not enough to eradicate bacterial biofilm from the catheters. On the other hand, the MBECs for amikacin against these study isolates were 1600, 8000, 3200, 400, 800, 400, and $800 \mu \mathrm{g} / \mathrm{ml}$ respectively and no any colony appeared on the culture counting plate. Statistically, there is high significant difference (P value $<0.0001)$ with MICs mean $(10.29 \pm 3.90)$ and MBEC mean (628.57 \pm 213.8$)$ as represented in Fig. 8.

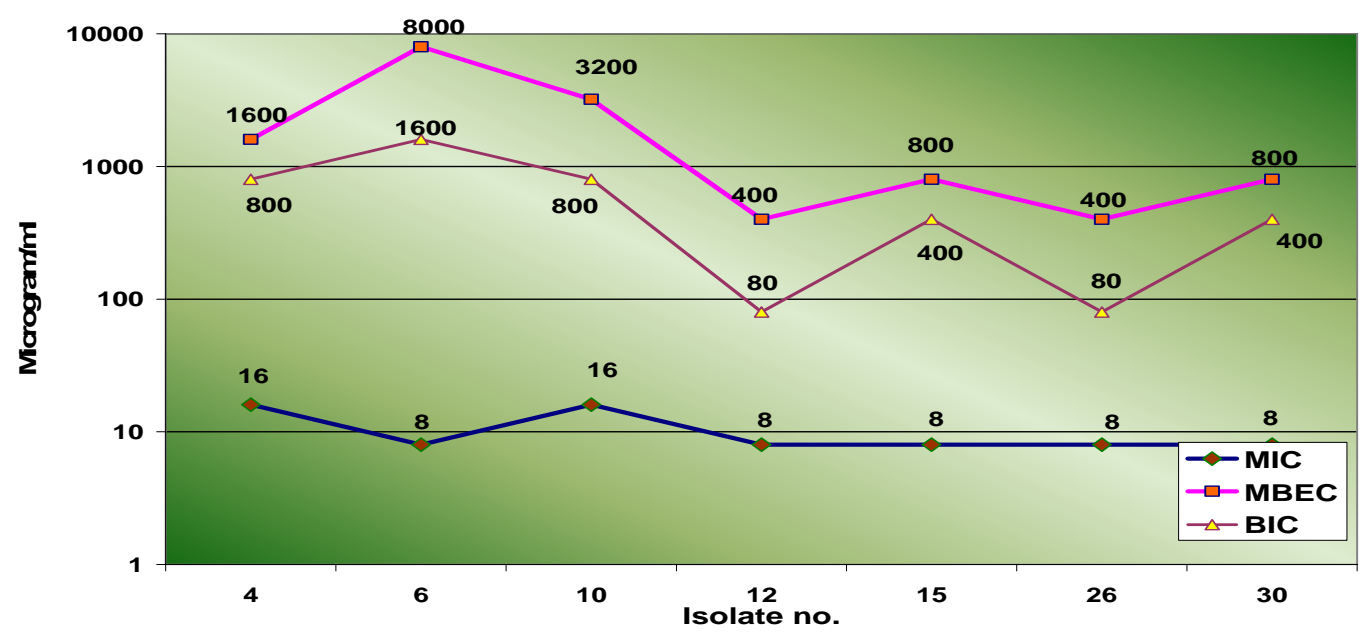

Fig. 8: Comparison of susceptibility parameters for amikacin against logarithmic phase planktonic cells and sessile (adherent) cells of seven study isolates of Pseudomonas aeruginosa. 


\section{DISCUSSION}

It is remarkable that the biofilm (adherence) mode of growth of bacteria on the surfaces of biomaterials has been well documented as one of the important causes of prosthetic device related infections that are refractory of antimicrobial therapy (Yassien and Khardori, 2001). Indwelling bladder catheters are the most commonly deployed prosthetic medical devices (Darouiche, 2001). Indwelling catheter constitutes a convenient means of managing bladder dysfunction; unfortunately, they also provide access for bacteria from contaminated external environment into a vulnerable body cavity. Implanted prosthetic devices constitute particularly attractive surfaces for bacterial colonization, as they have none of the protective mechanisms of health tissue surfaces. Unfortunately, the care of many patients undergoing long-term catheterization is frequently complicated by microbial infection. These microbial causes colonize the catheter, forming surface biofilm communities (Jones et al., 2005).

In the bacteriological part of this study, our study showed that Escherichia coli was the most common microorganism isolated from patients with indwelling urinary catheters followed by Klebsiella spp., Pseudomonas aeruginosa, Staphylococcus epidermidis, Staphylococcus aureus, Proteus mirabilis, Enterobacter aerogenes and Candida species respectively. On the other hand, (Talpur and co-workers, 2004) found that catheterization not only increased bacteriuria, but they also found that 92\% of prostatic tissues of catheterized patients were positive for bacterial cultures, while only $28 \%$ of prostatic tissues of non-catheterized patients showed positive bacterial cultures. The high rates of bacteriuria associated with catheterizations might be due to the presence of catheter bypasses normal host defenses allowing continuous access of microorganisms into the urinary bladder and markedly increasing the frequency of UTI.

Under the field of biofilm production, particularly qualitative adhesion assay, tube method, our result showed that out of 17 (27.9 \%) isolates of Escherichia coli, 13 (21.3 \%) isolates of Klebsiella species, 12 (19.7 \%) isolates of Pseudomomnas aeruginosa, and 7 $(11.5 \%)$ Proteus mirabilis, biofilm were produced on the inner lining of the tubes in 11 (64.7\%), 9 (69.2\%), 12 (100\%), and 7 (100\%) respectively. Our results are in agreement with those obtained from the study laid down by (Mathur et al., 2006) who observed good correlation between the results of qualitative tube assay and spectrophotometric assay regarding strongly biofilm producing isolates. The same authors concluded that it was difficult to discriminate between weak and biofilm negative isolates due to the variability in observed results by different observers. Consequently, high variability was observed and classification in biofilm positive and negative was difficult by tube method. Thus, tube test cannot be recommended as general screening test to identify biofilm-producing isolate.

In the quantitative biofilm formation assay, spectrophotometric method with ELISA reader was achieved under two set of experimental conditions (with and without glucose) and the crystal violet stain was dependent in this technique. In order to enable easier study of bacterial attachment and colonization, microtiter plate assay (spectrophotometric method) is the most frequently used techniques for quantifying biofilm formation (Stepanovic et al., 2000 ; Manzon et al., 2001). In this technique, our results showed that among 17 isolates of Escherichia coli, 12 (70.6\%) isolates were biofilm producers in the presence and absence of glucose. no significant differences observed in the readings of optical density at $570 \mathrm{~nm}$ in two conditions $(0.366 \pm 0.335)$ and $(0.351 \pm 0.282)$ respectively ( $\mathrm{P}$ value $=0.886)$. Also, 
among 13 isolates of Klebsiella spp, 12 (92.3\%) and 11 (84.6\%) isolates of this bacterium were produced biofilm in the glucose supplemented and non-supplemented media respectively. At other meaning, regarding these bacteria, adhesion process was not affected by the presence or absence of glucose in the culture media. (Anderson and colleagues, 2003) demonstrated that uropathogenic $E$. coli can form biofilms, albeit unusual ones. These authors showed that, while infecting the bladder umbrella cells, E. coli can invade into the cells and form biofilms intracellularly, consisting of small, coccoid-like cells. Protected by the mammalian cellular membrane, $E$. coli are sequestered from the phagocytic action of neutrophils. Intracellular E. coli may also be protected from antibiotic therapy. Eventually, however, the infected cell will apoptose and slough into the lumen of the bladder. During this process, the uropathogenic E. coli adds another twist - it changes morphology from small, almost coccoid cells to long, filamentous cells. This finding of intracellular biofilms, as well as being a description of a novel biofilm mechanism, also explains the persistence and recurrence of urinary tract infections (Dodge et al., 2006).

Also, it is well realized that the model of biofilm resistance is based on the persister's survival. An initial treatment with antibiotics kills planktonic cells and the majority of biofilm cells. The immune system kills planktonic persisters, but the biofilm persisters are protected from immune defense mechanisms by the extracellular matrix. After the antibiotic concentration drops, persisters resurrect the biofilm and the infection relapses (Vuong et al., 2004); (Lewis et al., 2006).

Antimicrobial susceptibility test for sessile cell of Pseudomonas aeruginosa was achieved by detecting the minimal biofilm eradication concentration (MBEC). The calculation of different concentrations of antimicrobial agents used was based on the minimal inhibitory concentrations obtained by broth macrodilution technique achieved against logarithmic phase planktonic cells of Pseudomonas aeruginosa. Our result revealed that the biofilm cells were required 50-100 times the MIC values for ciprofloxacin obtained for the same isolates in the logarithmic phase of planktonic cells with MIC mean (26.29 \pm 10.028$) \mu \mathrm{g} / \mathrm{ml}$ and MBEC mean $(1942.86 \pm 907.11) \mu \mathrm{g} / \mathrm{ml}$ with high significant difference ( $\mathrm{P}$ value $<0.0001)$. (Yassien and Khardori, 2001) documented that the high concentrations of flouroquinolones were used to treat the performed biofilms because 1) these concentrations would be expected to reach the biofilms when therapeutic doses of flouroquinolones (ciprofloxacin is one of the flouroquinolones) are infused through the vascular catheters and, 2) using such high concentrations would minimize the exposure of very large inoculum of bacteria in the biofilms to sub inhibitory concentrations of the flouroquinolones. This effect is concentration dependent. On the other hand, Lewis and associates, (2006) documented that resistance of biofilms to killing by antimicrobials is dramatic, 100-1000 fold above the MIC, and occurs for all antimicrobials tested and in all species examined. This means that all pathogens potentially harbor an essentially perfect multi-drug resistance mechanism, but only "choose" to express it when growing as biofilm.

It is well documented that the mechanisms of ciprofloxacin effect in the biofilm include: (1) electrostatic interference with the adhesion of bacteria and/or glycocalyx to the substratum, (2) activation or release of enzymes to disrupt the exopolysaccharide (glycocalyx) in the biofilm and, (3) inhibition of the formation of new glycocalyx. Irrespective of the mechanisms involved, the observed effects of the ciprofloxacin may 
improve the pharmacodynamics of the antibacterial agents used to treat prosthetic device related infections (Yassien and Khardori, 2001).

Also, with regard to cefotaxime, our result revealed that the biofilm cells were required 50-100 times the MIC values obtained against study isolates in logarithmic phase planktonic cells. (Mah and co-workers, 2003) explained the genetic basis for Pseudomonas aeruginosa biofilm antibiotic resistance by identifying a chromosomal locus in his study called 'ndvB' which his required for the synthesis of periplasmic glucans. These periplasmic glucans interact physically with antimicrobials and the formed glucose polymers may prevent antimicrobials from reaching their sites of action by sequestering these antimicrobial agents in the periplasm. Further, the same researchers indicated that biofilm themselves are not simply a diffusion barrier to these antimicrobials, but rather that bacteria within these microbial communities employ distinct mechanisms to resist the action of antimicrobial agents.

Also, with regard to amikacin, our result revealed that the biofilm cells were required 10-50 fold greater than MICs observed for study isolates of Pseudomonas aeruginosa in logarithmic phase planktonic cells. (Pascual, 2003) observed that MICs of amikacin against Pseudomonas aeruginosa increased at in the presence of siliconized latex, a biomaterial commonly used to make urinary tract catheters. This effect was not strain-dependent and was similar for different branches of catheters. In fact, this phenomenon was induced by substances eluted from the biomaterials (Pascual, 2003).

\section{REFERENCES}

Anderson, G. G.; Palermo, J. J.; Schilling, J. D.; Roth, R.; Heuser, J. ; Hultgren, S. J. (2003). Intracellular bacterial biofilm-like pods in urinary tract infections. Science., 301, 105-107.

Arif, S. K. (2006). Catheter associated bacteriuria and biofilm study. Ph.D thesis. College of Medicine, University of Al-Mustansiryia.

Baron, E. J.; Peterson, L. R. ; Finegold, S. M. (1994). "Bailey and Scott’s Diagnostic Microbiology". Toronto: C.V. Mosby Company. 9th ed.

Ceri, H.; Olson, M. E.; Morck, D. W. ; Storey, D. G. (2006). "Minimal Biofilm Eradication Concentration (MBEC) Assay: Susceptibility Testing for Biofilms". In: Biofilms, infection and antimicrobial therapy. By: Pace, J.L., Rupp, M. E. and Finch, R. J. Taylor and Francis, CRS Press, Parkway. 1st ed.

Costerton, J. W.; Stewart, P. S. ; Greenberg, E. P. (1999). Bacterial biofilms: a common cause of persistent infections. Science., 284, 1318-1322.

Darouiche, R. O. (2001). Device-associated infections: a macroproblem that starts with micro adherence. Clin. Infect. Dis., 33, 1567-1572.

Dodge, I. L.; Shaw, K. J. ; Bush, K. (2006). "ß-lactams for the Treatment of BiofilmAssociated Infections. In: Biofilms, Infection and Antimicrobial Therapy". By: Pace, J. L., Rupp, M. E. and Finch, R. J. Taylor and Francis, CRS Press, Parkway.1st ed.

Donlan, R. M. (2001). Biofilm formation: a clinically relevant microbiological process. Clin. Infect. Dis., 33, 1387-1392. 
Donlan, R. M. (2002). Biofilms: Microbial Life on Surfaces. Emerg. Infect. Dis., 8, 881890.

Ferraro, M. J.; Craig, W. A.; Dudly, M. N.; Ehopoulos, G. M.; Hecht, D. W. (2000). "Methods for dilution antimicrobial susceptibility test for bacteria that grow aerobically". Approved standard, 5th ed. NCCLS. 20, pp. 1-24.

Fux, C. A.; Stoodley, P.; Hall-Stoodley, L. ; Costerton, J. W. (2003). Bacterial biofilms: a diagnostic and therapeutic challenge. Expert. Rev. Anti-infect. ther., 1, 667-683.

Ishida, H.; Ishida, Y.; Kurosaka, Y.; Otani, T.; Sato, K. ; Kobayashi, H. (1998). In vitro and In vivo activities of levofloxacin against biofilm-producing Pseudomonas aeruginosa. Antimicrob. Agents. Chemother., 42, 1641-1645.

Jones, B. V.; Mahenthiralingam, E.; Sabbuba, N. A.; Stickler, D. J. (2005). Role of swarming in the formation of crystalline Proteus mirabilis biofilms on urinary catheters. J. Med. Microbiol., 54, 807-813.

Katsikogianni, M. ; Missirlis, Y. F. (2004). Concise review of mechanisms of bacterial adhesion to biomaterials and of techniques used in estimating bacteria-material interactions. Europ. Cell. Mat., 8, 37-57.

Lewis, K.; Spoering, A. L.; Kaldalu, N.; Keren, I. ; Shah, D. (2006). "Persisters: Specialized Cells Responsible for Biofilm Tolerance to Antimicrobial Agents". In: Biofilms, infection and antimicrobial therapy. By: Pace, J. L., Rupp, M. E., and Finch, R. J. Taylor and Francis, CRS Press, Parkway.1st ed.

Mah, T. F.; Pitts, B.; Pellock, B.; Walker, G. C.; Stewart, P. S. ; O’Toole, G. A. (2003). A genetic basis for Pseudomonas aeruginosa biofilm antibiotic resistance. Nature. 426, 306-310.

Manzon, M.; Oteiza, C.; Leva, J.; Amorena, B. (2001). Synergy of different antibiotic combination in biofilm of Streptococcus epidermidis. J. Antimicrob. Chemother. 48, 793-801.

Mathur, T.; Singhal, S.; Khan, S.; Upadhyay, D. J.; Fatma, T. ; Rattan, A. (2006). Detection of biofilm formation among the clinical isolates of Staphylococci: An evaluation of three different screening methods. Ind. J. Med. Microbiol., 24, 25-29.

Miles, R. S. ; Amyes, S. G. B. (1996). "Laboratory Control of Antimicrobial Therapy. In: Practical Medical Microbiolgy". By: Collee, J. G., Barrie, P. M., Fraser, A. G., Simmer, A. California, Churchill, Livingston, 14th ed.

Mims, C. A.; Playfair, J. H. L.; Roitt, I. M.; Wakelin, D.; Williams, R. (1993). Urinary tract infections. In: Medical Microbiology. Mosby.

Mireles, J. R.; Toguchi, A. ; Harshy, R. M. (2001). Salmonella enterica serovar typhimurium swarming mutant with altered biofilm forming abilities: Surfactin inhibits biofilm formation. J. Bacteriol., 183, 5848-5854.

Olson, M. E.; Ceri, H.; Morck, D. W.; Buret, A. G. ; Read, R. R. (2002). Biofilm Bacteria: formation and comparative susceptibility to antibiotic. Canad. J. Veterin. Res., 66, 86-92.

Pascaul, A. (2002). Pathogenesis of catheter-related infections: lessons for new designs. Clin. Microbiol. Infect., 8, 256-264.

Stepanovic, S.; Djukic, V.; Djordjevic, V. ; Djukic, S. (2003). Influence of the incubation atmosphere on the production of biofilm by Staphylococci. Clin Microbiol. Infect., 9, 955-959. 
Stepanovic, S.; Vukovic, D.; Dakic, I.; Savic, B.; Svabic-Vlahovic, M. (2000). A modified microtiter plate test for quantification of staphylococcal biofilm formation. $J$. Microbiol. Meth., 40, 175-179.

Stickler, D. J. (1996). Bacterial biofilms and the encrustation of urethral catheters. Biofouling., 94, 293-305.

Talpur, A. N.; Hasan, A. T. ; Sheikh, M. A. (2004). Intraprostatic tissue infection in catheterized patients in comparison to controls. J. Pak. Med. assoc., 54, 20-24.

Von-Eiff, C.; Jansen, B.; Kohnen, W. ; Becker, K. (2005). Infections associated with Medical Devices: Pathogenesis, Management and Prophylaxis. Drugs, 65, 179-214.

Vuong, C.; Kidder, J. B.; Jacobson, E. R.; Otto, M.; Proctor, R. A. ; Somerville, G. A. (2004). Polysaccharide intercellular adhesin (PIA) protects.

Yassien, M. ; Khardori, N. (2001). Interaction between biofilms formed by Staphylococcus epidermidis and quinolones. Diag. Microbiol. Infect. Dis., 40, 79-89. 